Document downloaded from:

http://hdl.handle.net/10251/51900

This paper must be cited as:

Sempere Paya, VM.; Santonja Climent, S. (2012). Integrated sensor and management system for urban waste water networks and prevention of critical situations. Computers, Environment and Urban Systems. (36):65-80. doi:10.1016/j.compenvurbsys.2011.07.001.

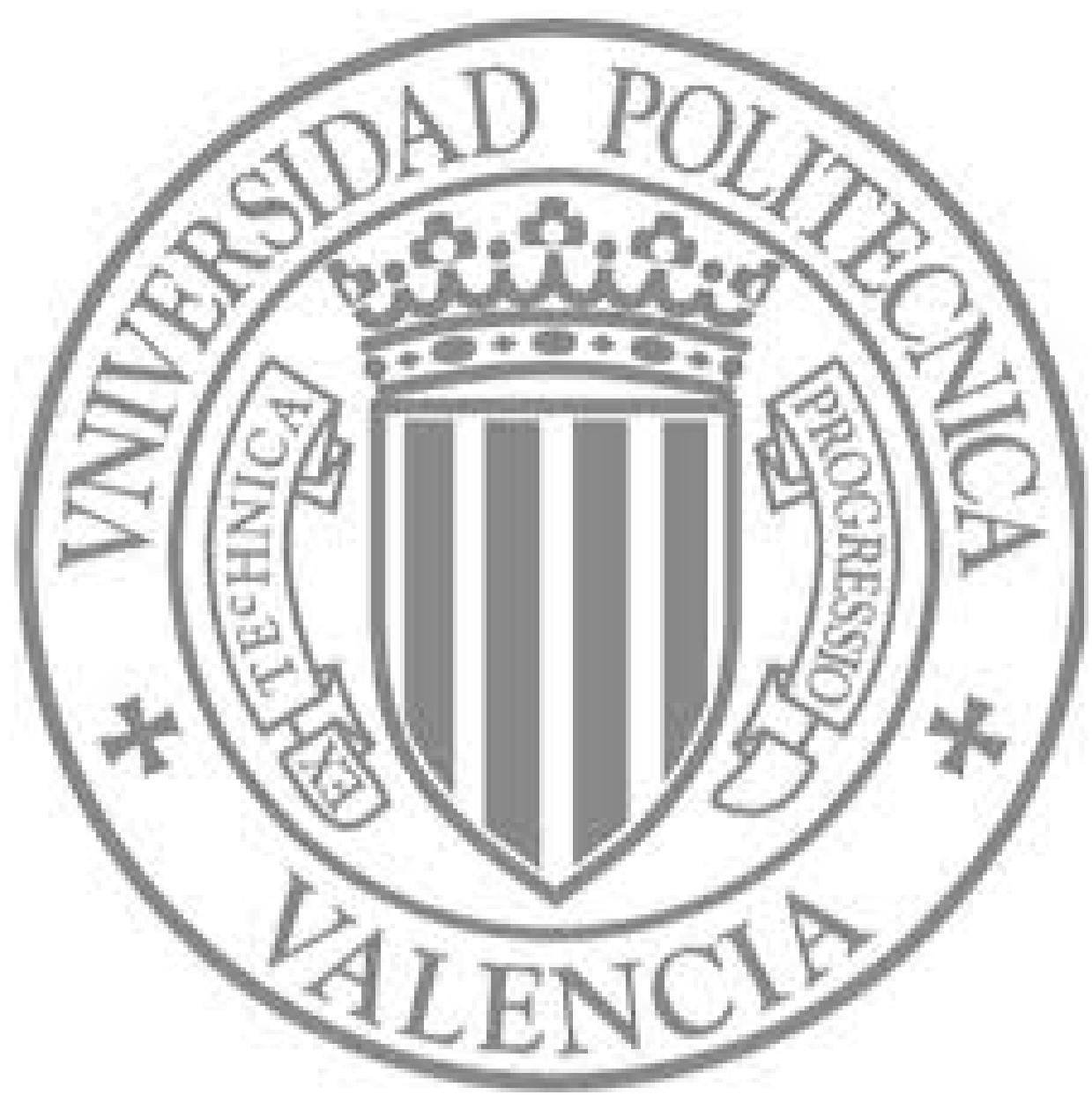

The final publication is available at

http://dx.doi.org/10.1016/j.compenvurbsys.2011.07.001

Copyright Elsevier 


\title{
Integrated sensor and management system for urban waste water networks and prevention of critical situations
}

\author{
Víctor-M. Sempere-Payá, Salvador Santonja-Climent \\ Instituto Tecnológico de Informática, Universidad Politécnica de Valencia, 46022 Valencia, Spain
}

\begin{abstract}
This work describes the design and implementation of improvements to the monitoring system of an urban waste water network, resulting in more efficient management of the system. To achieve this objective, the latest communications technology has been incorporated into heterogeneous networks and sensor systems. This technology includes mobile systems, which take measurements and transmit images in real time, an intelligent platform for processing and management of variables, and the implementation of wireless sensor networks (WSNs) designed with specific protocols and tools that allow the rapid deployment of the network and allow measurements to be taken in emergency situations. The sensors in this type of installation are extremely important for the management of the system as they allow us to collect information and make decisions with sufficient time to deal effectively with critical situations such as flooding or overloading of the waste water system, or environmental problems such as dumping of possible pollutants, as well as to make the best use of the water cycle. The solution presented here automates large portions of the processes, minimizing the possibility of human error, and increasing the frequency and accuracy of the measurements taken, ensuring a robust communication system covering all the elements involved to provide ubiquity of information, and finally gives an application layer to manage the system and receive alerts.
\end{abstract}

Keywords

Heterogeneous networks, water cycle, Utilities Networks, Wireless Sensor Networks (WSN), Machine to Machine (M2M)

\section{Introduction}

The waste water systems of large cities manage, among other things, the drainage of rain water and the transport of waste water prior to treatment. These services may pass unnoticed by the city's inhabitants as the responsible departments work intensively, day to day, using specific protocols to deal with critical situations. However, a lack of information and coordination when facing unpredictable situations, as well as insufficient time to prepare any necessary action, can quickly result in chaos due to flooding and overflows. This is the case in the city studied here, the Spanish city of Valencia, which has a Mediterranean climate, generally warm and with annual rainfall of around $484 \mathrm{~mm}$. However, due to the extra-tropical phenomenon known as "High Level Isolated Depression" (HLID), rainfall is usually concentrated in the autumn months in the form of very aggressive storms of short duration with extremely intense rainfall (which can surpass those found in inter-tropical areas) (Fig.1). This phenomenon makes preparation and prevention tasks, rain water drainage, and management of the system, extremely difficult. In the late 1900 s, there were many disasters due to flooding in the city, which highlighted the need for improvements in the sensor systems of the waste water network to ensure a rapid response in terms of operating and managing the network during critical situations in the future.

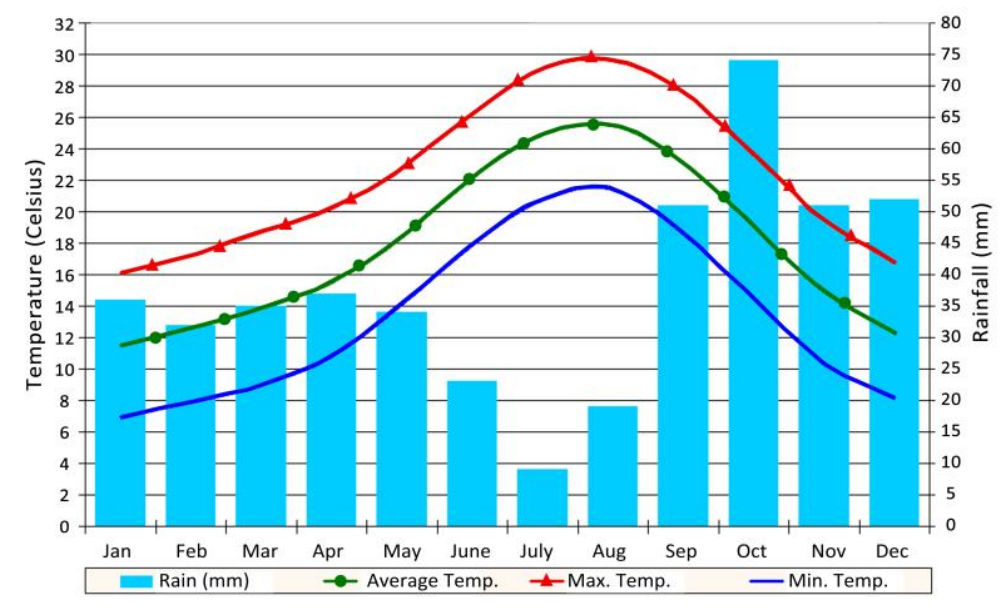

Figure 1. Average rainfall in Valencia between 1971 and 2000. Spanish State Agency of Meteorology.

Waste water management also has a fundamental role in the environmental contribution of the city, as there are a range of actions and operations to be carried out before the water is released into the sea. It is vital to make the best possible use of the 
water cycle through purification and the use of large subterranean tanks which collect rain water run-off, and it is also important to manage the drainage system and control water quality before it is released into the sea.

Whether it be for environmental reasons or for the safety of the population, it is important that periodic measurements are taken from the system to control environmental parameters, as well as water quality and flow in the drainage water courses. Until now, these tasks have been carried out by a series of Environmental Control Stations (ECSs) distributed throughout the city. Many of these stations are in locations with communication network access problems, meaning that workers have to travel from station to station to collect data. There are also points where there is no fixed measuring infrastructure, and so it is impossible to obtain the necessary information. This has meant that on many occasions, when a problem has been detected, it is already too late to react effectively. High risk situations cannot be predicted with enough time in hand, and the quality and frequency of data collection is inadequate to operate a solid prevention system.

Thus, it became necessary to integrate an intelligent sensor system which provides new tools and processes to give added value to the management system of the city's waste water network. This article describes the technological infrastructure necessary to capture and transport information. We also show how making use of the latest information and communications technologies (ICT) gives us ready access to high quality information at critical times. Finally, we provide results of a performance evaluation carried out from the point of view of a service user, in order to understand the user's perceived performance and the level at which the system provides necessary services.

The structure of the remainder of the paper is as follows: Section 3 presents a global description of the system developed, defining the various parts which make up the system as a whole and also how they are connected. Sections 4, 5 and 6 detail the need for the remote monitoring system, as well as a description of the complete system and the results obtained after its implementation. Section 7 explains the Central Control Station (CCS) where the flow of information is controlled and analyzed, as well as how it performs with a human-machine interface (HMI) system. This is followed by conclusions in Section 8 .

\section{Related Work}

Urban waste water networks depend largely on personnel for daily management and operation, although there are some minor automation tasks based on old polling systems for monitoring purposes that limit the response and location of the stations. In recent years, developments such as real-time control (RTC), homogenization, increased sensory capacity of waste water networks, and automation, have been key factors in contributing to the sustainable development of cities and their greater commitment to the environment. Water management projects such as those undertaken in Quebec (Canada) (Schütze et al., 2009), Emscher/Lippe (Germany) (Verworn, 2002), or Barcelona (Spain) (Ocampo et al., 2009), integrate fully automated systems with telemetry and telecontrol elements to provide real-time control over the network. These projects demonstrate ways in which the performance of the drainage system can be improved by generating flow control strategies and better water use strategies. A key aspect of the modernization of these networks is the adoption of Decision Support Systems (DSS) to aid in the rapid response to a disaster situation. The Neptune project (Morley et al., 2009) of the University of Exeter, and Blue Box Tool (ACQUEAU, 2010) from Whitewater (Israel) are two examples that focus on monitoring water quality, prevention and early action in case of contamination. The UrbanFlood project (Pyayt, Lang, Mokhov, \& Ozhigin, 2010) of Ijkdijk city in The Netherlands uses a sensor network on dykes for the early prediction of structural breaks and floods, as well as management and decision making assistance. COW AMA (Suñer, Malgrat, Leitão, \& Clochard, 2008) is a decision support system for the management of bathing water. With the entry and flow of water into the sewers, direct discharges of sewage to the receiving environment, sewage treatment and dispersal in the sea, this system can predict the magnitude and duration of pollution episodes.

Many cities are still reluctant to implement RTC and DSS systems, mainly due to the difficulty of design and the level of development of sensors and communications systems ${ }^{1}$. Establishment of a complete urban sensing network is complex and expensive, and it is difficult to provide adequate cover. This project provides one step in overcoming some of these obstacles by illustrating a real case for improving an urban waste water system through the integration of traditional environmental monitoring stations and a new component of mobility: environmental monitoring laboratories and rapid deployment sensing

\footnotetext{
${ }^{1}$ In January 2004 the UN Secretary-General called for Global Early Warning Systems (EWS) addressing all natural hazards. In his 21 March 2005 Report on the implementation of the Millennium Declaration, «In Larger Freedom: towards development, security and human rights for all», he requested the secretariat for the International Strategy for Disaster Reduction (ISDR secretariat) to coordinate a survey of the world's early warning capacities and gaps. Preliminary findings highlight the need of better sensor and transmission systems for early detection of these disasters.
} 
networks that assists staff during installation. These elements can be placed anywhere and provide information to the CCS in real-time with minimal cost, all of this integrated in a homogeneous communications network, accessible from anywhere. Mobility block is proposed as a new powerful toolkit for urban waste water network management, enabling managers to go beyond the limits of the existing fixed monitoring networks, and reduce the costs of installing new stations in the system. Reusability and ease of deployment of such networks enables the installation of a fully functional sensing system anywhere it is necessary and in a short period of time, favouring rapid data acquisition for better decision making in critical situations (flooding, contamination, breakages, etc).

\section{Diagram of the advanced management and sensor system}

Improvements in the monitoring and control system of a city's waste water system enhances the performance and operability of existing infrastructure, and the creation of new remote measuring systems increases sensor capacity and substantially improves the system's response to critical situations (mainly introduction of foreign material to the water courses and intense rainfall). The efficiency of the system as a whole is highly dependent on communication between the component parts, as well as the link with the Central Control Station (CCS) and their automation. The structure of the system can be seen in Figure 2: the elements of the sensor system inform the Central Station of the current situation via a heterogeneous IP communications network (Andritsou, \& Pronios, 2001). These sensor elements are grouped into fixed stations which obtain environmental data, water quality parameters, and phreatic levels at critical points around the city (ECSs and piezometers); mobile Units for Residual Water Quality Control (UCCAR), which can be located at secondary points, reducing the need to install fixed stations and reducing maintenance costs; and the Quick Deployment Sensor Networks (QDSN), which allow occasional monitoring of areas of interest in a quick precise way. In the CCS, the management system evaluates the data received to then inform the personnel responsible, or makes decisions on any action that needs to be taken. The operators who are physically in the CCS can access all information, both past and current, and manage the system. They can also access any necessary images from the network to use as visual support to help with decision making. Moreover, the web platform gives controlled access to remote clients located outside of the CCS, which makes managing the system even easier for personnel.

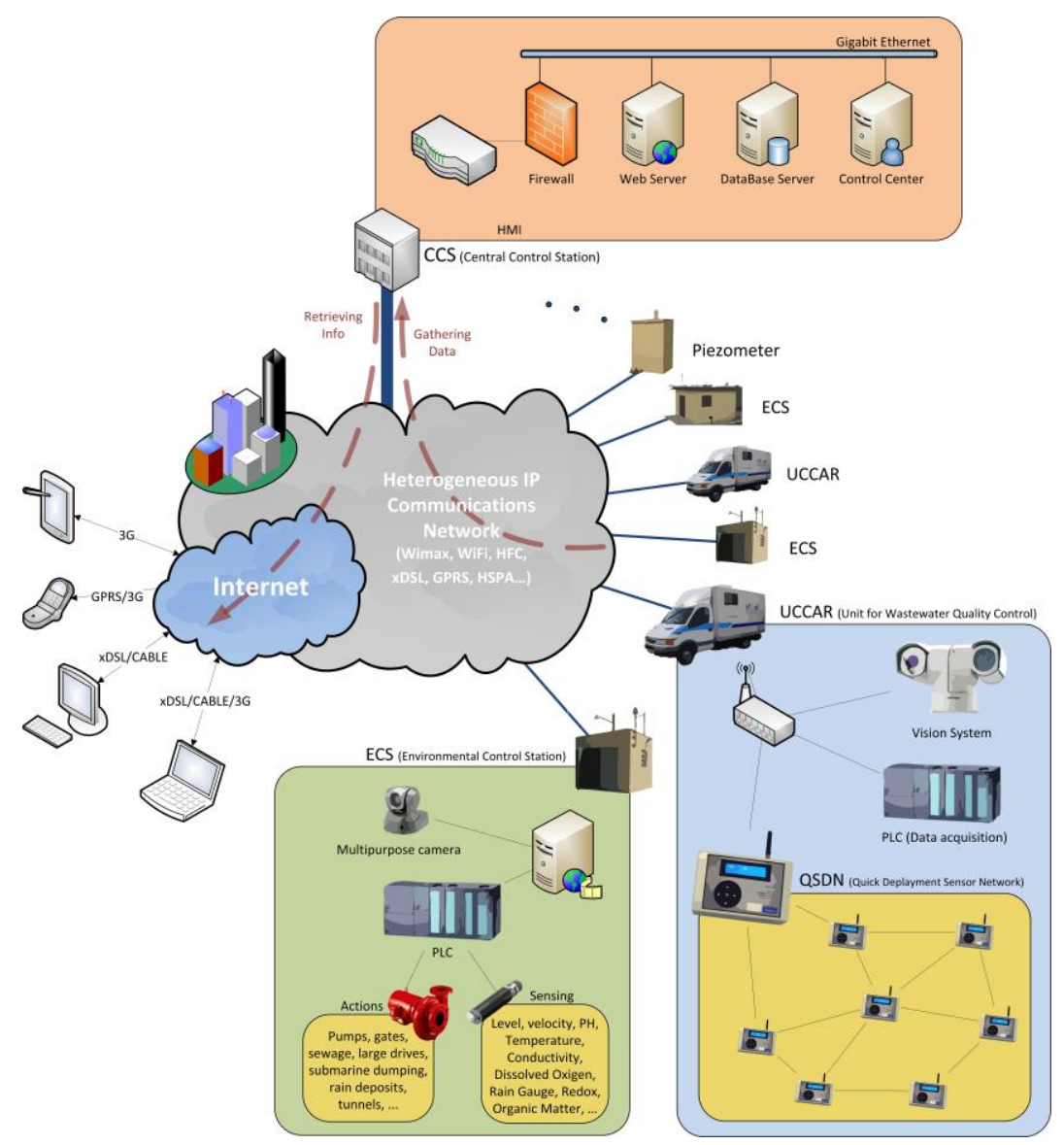

Figure 2. Environmental Data Telecapture Architecture 
The advanced sensor system in waste water networks allows, among other things:

- Knowledge of the state of the entire network in real time.

- The ability to warn of or prevent emergency situations (such as detecting pollutants dumping in industrial areas before it arrives at city sewage or water resources).

- Water courses to be modeled which establish the dimensions of storage and rainwater tanks.

- To determine if new infrastructure is needed

- Modeling the impact of dumping on natural water courses.

- Feedback to contrast the model data with real data.

In the following section, we describe the architecture of each of the parts which make up the sensor structure: the fixed stations (ECS), mobile stations (UCCAR), quick deployment sensor network (QDSN) and the Central Control Station (CCS).

\section{Fixed Station Architecture}

\subsection{Objectives and requirements}

The fixed measuring stations incorporate sensors to capture environmental and water control data (Ritchie \& Cooper, 2001). Of the sensors which are usually used, we can highlight those for ultrasonic level measurements, doppler velocity, area-velocity, level by bubble, PH-temperature, conductivity, dissolved oxygen, rain gauge, suspended soil, organic matter, redox probe and the piezometers. There is also equipment to take samples automatically (samplers), which collect water samples when a parameter that is outside recommended levels is detected. This sample is later analyzed in the laboratory. The system must alert the technicians so that the samples are processed within 24 hours so that the samples are still valid.

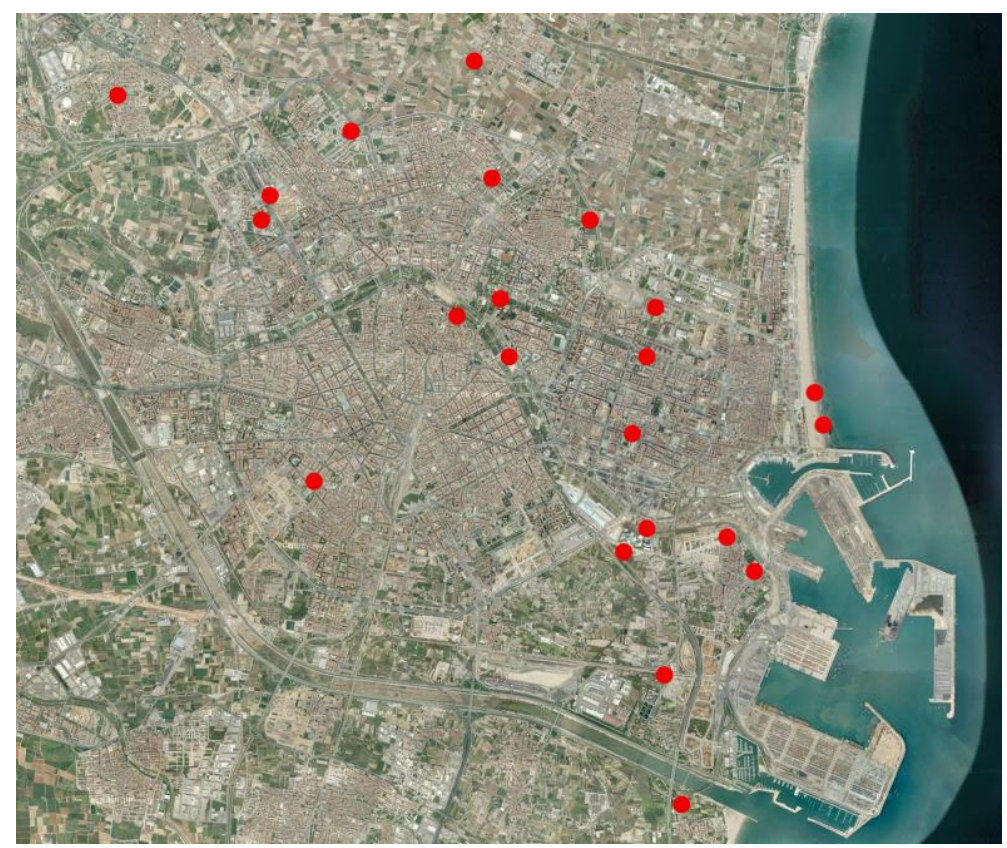

Figure 3. ECS Distribution in Valencia City

Fig. 3 shows the distribution of the ECSs (Environmental Control Stations) in the metropolitan area of Valencia. This area covers $69 \mathrm{~km}^{2}$, where the range, cost, and heterogeneity of devices and protocols of the communications systems (many stations lack the possibility for connection to fixed communication networks) has, until now, created great difficulties for its automation. This has meant that technicians have had to physically visit all the ECSs with a laptop computer, together with the software provided by the data acquisition system manufacturer (datalogger) to download the information collected by the sensors. Often, software and hardware made by different manufacturers are present in different stations which creates a great deal of inconvenience for the technicians and often means complex tasks are necessary to integrate the information. These tasks are carried out once a month, or in periods of heavy rainfall when alert reports are necessary. The time needed for the number of ECSs with the distribution shown in Fig. 3 was between 4 and 8 hours, depending on parameters as variable as the level of traffic in the city. 
To resolve the problems described and to be able to access information quickly and easily, it is necessary to develop a plan in which:

1. The ECSs capture data from geographically dispersed locations through open architectures and interfaces, thus developing systems that are more flexible, economical, and which offer scalability and are easy to operate (Granzer, Kastner, Neugschwandtner, \& Praus, 2006).

2. The measurements are collated at a central point, the CCS, using standard communication technologies and protocols, and where complete control of the network can be obtained (Thomesse, 2005).

3. Using this architecture, in the CCS it will be possible to develop a data management system with open software that is not tied to the equipment manufacturers.

\subsection{ECS Architecture}

The objective of the architecture is to homogenize the wide range of devices available on the market, thus avoiding the problems described above. To obtain a system with the best possible compatibility between sensors with different manufacturers, and to give greater flexibility in programming and communication, we have used a micro-PLC (programmable logic controller) as the core element of the fixed stations. The connection with the probes is carried out using $\mathrm{I} / \mathrm{O}$ analog modules of 4-20 $\mathrm{mA}$ and digitals with the sampler using a digital output activated by relay. The interface with the communications network is carried out using a Gateway or appropriate modem for the access technology in each station, in such a way that the communication process is homogenized.

\subsection{Communication architecture}

There are three traditional architectures that can be used to control communications between ECSs and the CCS:

- $\mathbf{S}$ (Synchronous). The information is transmitted periodically. The ECS transmits information on the state of the variables at predefined regular time intervals.

- $\quad \mathbf{R}$ (Request by central). The information is transmitted when explicitly requested by the Central. The ECS transmits observations on the state of the variables when this information is requested by the CCS.

- $\mathbf{A}$ (Asynchronous). The information is transmitted when triggered by state changes. Changes in the state of the ECS will generate the transmission of the corresponding notification from the ECS, so that the CCS can register these changes and initiate any action that may be necessary depending on the alert received.

The choice of model is determined by various factors, such as:

- The communication network, the services and the tariffs offered by the operator.

- The capacity of the ECS and CCS.

- The requirements of the application that is using the information.

\subsubsection{Application requirements}

From the point of view of the application, a single model of events such as those described in the previous section is inadequate and insufficient. Although it is necessary to have periodic access to information, it is also necessary to have a nonperiodic model which is able to transmit alarms when a value outside of the normal range is recorded. It is also important to be able, from the CCS, to request a reading of current values, independently of the other two methods, so that the technicians have the information they need available to them when necessary.

The pure synchronous communication model $(S)$ would mean an unnecessary overload of transmissions which, depending on the communication service in question, could have extremely high costs. Moreover, it is not viable to determine a capture and transmission frequency that is appropriate for all the types of variables that must be transmitted from an ECS, as each of them has its own dynamics, and they are not always correlated. This makes it very complicated to base transmissions only on this method. Fig. 4 shows an example. From an environmental variable var1, two samples are taken, sample 1 and sample 2 , and from another environmental variable, var2, sample 3 and sample 4 are taken. These are captured after set intervals of time $t$. The figure also shows the values of the averages every $10 t$ (average1) and $20 t$ (average 2 ) of these same values shown every $t$. If the average values captured every $20 t$ were transmitted, the anomaly in the var 2 values would be imperceptible and almost impossible to detect in var1. Reducing this value to $10 t$ would ensure the detection of the anomaly in var2, but that of var 1 would 
remain barely perceptible. Transmitting with a period of $t$ var 1 , and $10 t$ var2 makes the information transmission programming very complex in the ECS. Transmitting everything with a period of $t$, when there are many variables which are much slower, would lead to the transmission of a great deal of redundant information for many of the variables.

The communication model (R) does not allow the automatic generation of alarms from the ECS to the CCS, meaning that the CCS would not perceive an emergency situation until it requested information from the ECS of the state of its sensors, which in a polling system would depend on the polling cycle time. The quantity of transmissions would be decided by the number of requests from the CCS, which, as in the case of $(\mathrm{S})$, could mean significant cost increases if the system is to be constantly updated.

The asynchronous communication model (A) is the most appropriate from the point of view of optimizing communication resources and costs. The information is only transmitted when the variable surpasses a predefined alarm threshold. For example, in Fig. 4 we can configure the system so that for var1, a transmission would only be initiated if the value 4.5 is surpassed, or for var2 if 3.5 is surpassed. However, from the point of view of managing the installations, we wish to know not only the variables that are within the normal limits, but also the evolution of these signals, and that these are stored in the database in case an operator wishes to consult them and generate reports.

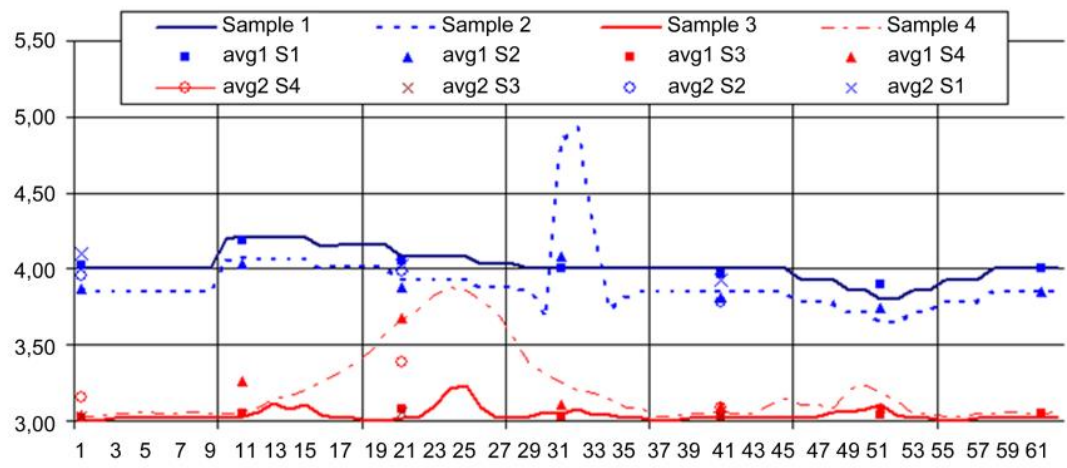

Figure 4. Samples

\subsubsection{Selection of communication models and the technology in use}

To satisfy the needs described in the previous sections, we have selected a hybrid model. Instead of sending the measurements exclusively with the model $(\mathrm{S})$, which would mean very short periods between transmissions to ensure early detection of any variation, a measurement is taken every second and is stored in the ECS itself, which then calculates a cumulative average over 5 minutes. After 5 minutes, this average value is transmitted to the CCS according to (S). This time gives us access to an adequate history of measurements to evaluate the behavior and evolution of the variables, but does not allow detection of instantaneous changes. For this second case, a series of thresholds is maintained, so that if one of the sensors surpasses one of the marked limits, a sample is automatically taken and an administrative alert is generated which informs the technical team of an anomaly in the measurements, as per communications model (R).

When choosing the access technology, one obstacle is that the ECSs are often located in zones with poor access, meaning that reaching them with a fixed network could be difficult and expensive. Tests with different licensed and open band radio systems have also given fairly negative results, with connections that fail repeatedly for long periods, or which depend closely on external variables such as meteorology, traffic or port activity. For this reason, and as the volume of information to be exchanged is not high (around 150 bytes for ECSs with 8 sensors), we chose GPRS (Sempere, Albero, \& Silvestre, 2006; Mayer \& Taylor, 2002). This technology gives coverage of the entire Valencia metropolitan area, and as it is mobile technology, it allows stations to be moved or mobile units to be incorporated into the system (see section 5).

\subsubsection{Communications service}

\subsubsection{Service for the ECSs}

The use of ordinary services to send data over a mobile network is not appropriate for these types of applications, as they are designed for high transfer rates and often there are service quality problems (Artell, Koivisto, Seppälä, \& Ruohonen, 2005). The data sent depends on the state and availability of the network, and there are no mechanisms to guarantee delivery or minimize delays. However, with the proliferation of small sized remote equipment that needs to be connected to the network for remote 
measuring or remote control systems, service providers have realized the need to design and market products which meet the specific needs of these systems. Because of this, M2M (Machine to Machine) services have appeared, which have real advantages over the traditional lines, including:

- Security: control of access to the network M2M, transport of coded and authenticated data.

- High availability: via a network that is optimized for M2M environments, configured for redundancy and backups, which means that the traffic generated and received by M2M devices arrives without errors.

- Flexibility: offers all the communication possibilities available (HSPA, UMTS, GPRS, Data GSM...).

- Specific incident and maintenance service.

- Scalability: allows a stock of SIM cards which are not invoiced until they are used for the first time.

- Added value: depending on the operator, various services are offered, such as creation of groups, Broadcast and Multicast transmission, notice of delivery, GSM location, presence control at the terminal, time synchronization, etc.

Equipment with M2M services connects to an Access Point Name (APN) different from that of normal lines, accessing a parallel network which manages the network traffic and guarantees quality of service.

\subsubsection{Service for the CCS}

The server that communicates with the elements of the M2M network can be accessed openly via internet or via a contracted public intranet network service, which offers significant benefits (Hopkinson et al., 2010):

- Security and privacy: data protection is guaranteed as it cannot be accessed by standard internet.

- Private addressing scheme: all of the M2M network becomes a LAN with its own choice of private addressing, while with public access to the internet the remote elements use public IPs which:

- In the case that they are dynamic, would require the use of dynamic DNS services (Östmark et al., 2006) for the use of mnemonics instead of IP addresses, or "Keep Alive" messages between server and clients to maintain the connection in use and thus not lose their public IP, which would incur additional expense.

$\circ$ In the case that they are static, they would incur an additional charge (60 € per IP line, monthly).

- Less traffic: The traffic in the GPRS stations is limited to a monthly quantity. If this limit is reached prematurely, there is an extra charge for new data. The consumption of the lines can be theoretically calculated so that it does not reach this limit, but open use of the internet means continuous traffic (search bots, external attacks, management systems, etc.) which also count as station traffic. With an intranet, this traffic does not reach the stations, only local traffic that is generated internally does.

The intranet is formed through the creation of a Virtual Private Network (VPN), via a standard internet connection (DSL, Cable) + IPSec. Using IPSec requires a RAS (Remote Access Server) for the authentication and assignment of client addresses (The remote stations) and an IPSec terminator. Using a purchased RAS server eliminates the need to rent one from the service provider. With this configuration, the remote stations, M2M clients, are connected to the RAS server when they access the data service, and after their authentication they are assigned an IP from the private selected addressing scheme. The data moves securely around the network and all the elements of the network know the addresses of the rest.

\subsubsection{Service costs}

The cost of the intranet service for M2M with the service provider chosen is $150 €$ per month, regardless of the number of stations to be connected. The CCS access to the internet gives no additional costs as it uses redundant high speed access that already exists. On the other hand, the internet access cost for M2M clients is a monthly fee plus a fixed traffic fee, as well as a separate price if the traffic limit is exceeded. If each station transmits a maximum of 150 bytes every 5 minutes, 24 hours a day, this gives a maximum monthly consumption of 1.24 MBytes, which, allowing for additional re-send traffic and a safety margin, means it is possible to contract a fee for 2 Mbytes per month. With this consumption and the number of lines in the project, we chose a service with a cost per line of $1.5 €$ and $2 €$ traffic fee, which comes to $42 €$ per year per line with traffic and maintenance included.

$$
\text { Total cost }=(42 \times \text { number of stations })+150 \quad[€ / \text { month }]
$$


With the system implemented here, the frequency with which environmental and water quality measurements are obtained has been increased from once a month to once every 5 minutes. The collection of physical samples in the ECSs when the values surpass a particular threshold is done the same day, thanks to the warning that is transmitted to the CCS. Therefore, chemical parameters don't have time to change and the sample is completely valid for laboratory analysis. Loss of connection with the stations is virtually nil, even during storms, which are the most critical periods for the system. The quantity of measurements taken is much higher ( 1 measurement every second, with an accumulated average every 5 minutes), which gives much greater precision in the control of the waste water system. Due to the scalability of the system, new ECS stations have been incorporated into the system. The information captured involves fewer resources than before and is therefore cheaper, and also the number of measuring points has increased.

\section{Mobile ECS architecture}

\subsection{Definition}

The mobile Units for Residual Water Quality Control (UCCAR) incorporate sensors similar to those of the ECS (flow velocity, level, conductivity, PH and temperature, organic matter, suspended soil, ammonia, sampler), but are not limited by being tied to only one measuring point. The transport system is a mobile laboratory vehicle which can be located wherever necessary to carry out water analysis. With this vehicle it is possible to have an environmental station in locations where it is not viable or beneficial to maintain a fixed station, such as terrain where is not possible to build a housing for the equipment (environmental laws, property rights). Moreover, the UCCAR also has two cameras to control the drains: the first camera is motorized and is introduced into them to monitor the interior, checking for blockages, cracks or other anomalies; and the second is used to inspect drains without the need to physically enter them, by introducing the camera into the surface openings of the drains. In both cameras, recording is done using an external hard drive, which is later taken to the CCS for analysis of the images.

\subsection{Integration of measurement system}

To incorporate the sensors in the CCS, and thus include the actively measured data in the system, a system similar to that the ECSs but with some characteristics derived from their mobility was used:

- The time marks of the samples taken are very important when they are later analyzed in the CCS, and so the system clock will be synchronized with the atomic clocks of the GPS satellites, which gives more than enough precision for this system. The system uses UTC +0 without the daylight saving hour change.

- The geo-reference mark is obligatory, due to the mobility of the system. The data received in the central station can only be correctly interpreted if the zone in which it was gathered is known.

Each frame will incorporate a time mark and a geo-reference in UTM-ED50 format (standard in Spain), as well as the measurement data. If the system is not positioned correctly (the GPS signal is not valid or there are not enough satellites to obtain it), the measurements will still be sent, but it will activate a "value not valid" bit, so that it is correctly processed in the CCS (the data from the sensors is correct, but the place in which they were obtained is unknown).

In this way, the mobile stations will be treated as simply one more station by the CCS, with one piece of information added which is the geo-reference (fig.5), so that they can be processed by GIS (Geographic Information System). The hardware and communication system are those described in the section "Fixed stations".

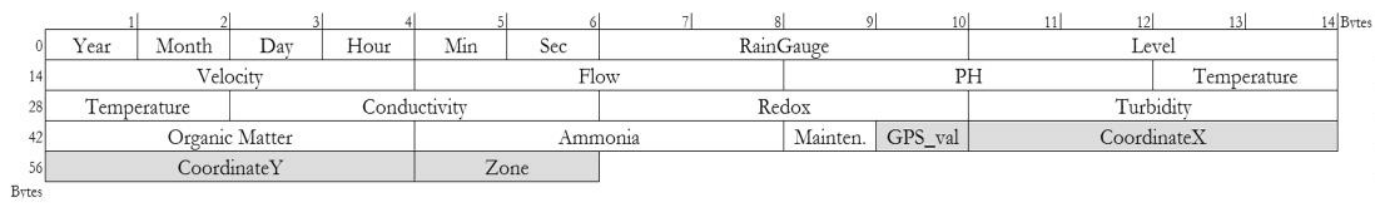

Figure 5. Uccar Data Frame, 62 bytes

\subsection{Improvement and integration of vision system}

The vision capabilities of a mobile station are an efficient mechanism to detect and prevent problems in the water. Through image capture it is possible to detect breaks, blockages, increases in flow, dumped material and suspended solids. To improve its performance, three updates have been carried out (fig.6): 


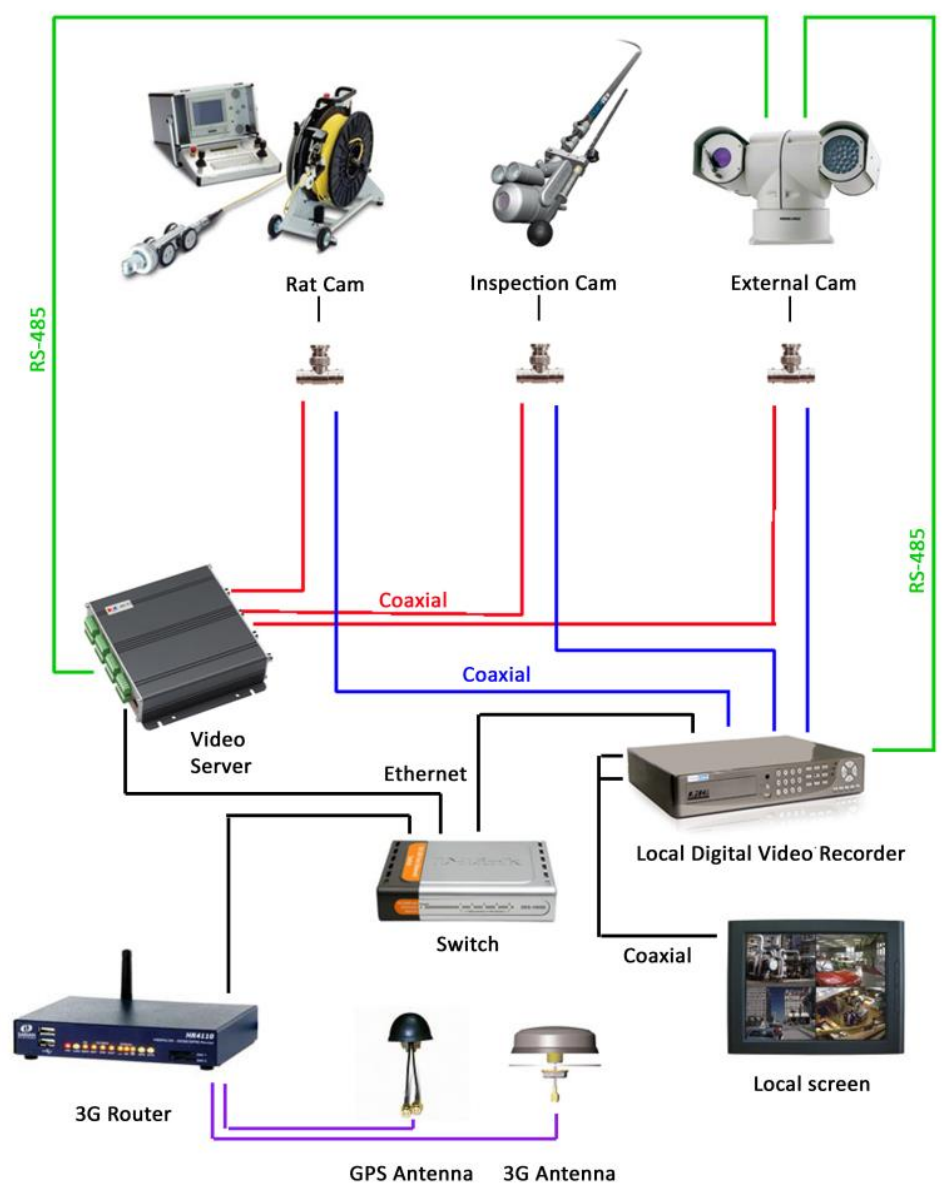

Figure 6. Video transmission system in UCCAR.

- An external camera has been added, which is fixed to the vehicle. With this camera, it is possible to station the UCCAR at a point where a particular event is to be monitored, for example spillways of the sewage system, or observe a zone which may experience dumping.

- Integration of the vision system is the only local system for control, management and recording.

- Real time communication between the vision system and the CCS.

\subsubsection{System integration}

Integrating the three cameras is done using a digital video recorder (DVR) with movement control, connected to a monitor. In this way, it is possible to choose a channel to observe, move the upper camera, and carry out configurations (wipers, infra-red, de-misting heater, etc...), recordings on the internal hard disc, movement detection and other advanced configurations. The compression is done with H.264 (Wiegand, Sullivan, Bjntegaard, \& Luthra, 2003), which gives the system autonomy for 1033 hours, equivalent to 46 days of continuous recording over all the channels simultaneously, at maximum quality.

H.264 is currently the most efficient video compression technique, allowing storage of high quality images while taking up less space on the hard drive. This is an evolution of the MPEG-4 system and has been specifically developed to give better video quality with a lower bit rate than other systems, using advanced techniques such as coded block patterns (which predicts movement in video frames).

\subsubsection{Communications system}

\subsubsection{Selection of technology}

Communication with the CCS introduces an important challenge due to the need to transmit three high resolution flows simultaneously from any point in the city. The first step is to code them. As the cameras are analog, a simultaneous multichannel video server is the best option, with H.264 coding and IP transmission. This server generates a bit rate of $330 \mathrm{Kbps}$ 
when transmitting only 1 channel at Full-D1 (720x576 pixels) with a frame rate of 1 per second and a fixed coding rate of 256 Kbps, and of $1 \mathrm{Mbps}$ with the 3 channels simultaneously. This involves using an uplink channel with high capacity in wireless communications, which means using, depending on availability, private (IEEE 802.11n) and public wireless technologies (UMTS, Universal Mobile Telecommunications System) (Stockhammer, \& Hannuksela, 2005).

In the city of Valencia, High-Speed Uplink Packet Access (HSUPA) is the most advanced public wireless technology, and it also has sufficient coverage. HSUPA is specified in the UMTS release 6, and is an evolution of High Speed Downlink Packet Access (HSDPA) which is focused on improving upload speed through the use of an enhanced dedicated channel (E-DCH) in the upstream. Using this technology, it is possible to achieve rates of 7,2 Mbps of downlink and $2 \mathrm{Mbps}$ of uplink, giving an improvement of 5 times the uplink rate of its predecessor. The UCCAR, which generates content for the network, will use this improved uplink channel. With this new rate of transfer, it is theoretically possible to transmit one video flow at a rate of 1,95 Mbps with a frame rate of $25 \mathrm{fps}$ and a coding bit rate of $1500 \mathrm{Kbps}$, or 3 simultaneous flows with a frame rate of $5 \mathrm{fps}$ and a coding bitrate of $500 \mathrm{Kbps}$, which significantly improves the quality of the system. It is important to remember that all these data are theoretical, and that the video transfer rates of the real system are conditioned by factors such as coverage, distance from the antenna, capacity and quantity of users, environmental factors, interference, etc. Therefore, the configuration of the video server is kept at dynamic bitrate, in such a way that it adapts the coding rate to the uplink bandwidth of the channel. The frame rate is maintained at $25 \mathrm{fps}$ and the resolution at 720x576 pixels (Schierl, Wiegand, \& Kampmann, 2005).

Here, contracting an M2M service would not be adequate, as the video traffic is much greater and it is sufficient to use a "Best Effort" service, and therefore, we use a standard data traffic service with a limit of 5 GB at a cost of $29 € /$ per month.

\subsubsection{Hardware}

The router chosen works with HSUPA technology, and is able to commute to other inferior technologies (such as HSDPA, EDGE or GPRS) if there is no coverage. Moreover, it has slots for two SIM cards (Subscriber Identity Module) from different operators, and in this way, the router can choose the operator to connect to depending on the coverage in the area. It is compatible with the Virtual Router Redundancy Protocol (VRRP) and VRRP+, which means it can coexist with a WiFi router, and through this protocol manage the vision system connection via IEEE $802.11 \mathrm{~b} / \mathrm{g} / \mathrm{n}$ networks (which has cost, speed and quality advantages) or via public networks. It incorporates a GPS receiver too (Global Positioning System) for the georeferencing of the images. Both the DVR and the video server flows are connected to the router. In this way, the DVR can be remotely accessed to view recordings from the CCS, or to operate the system as if the member of CCS personnel were present in the vehicle.

\subsubsection{Results}

\begin{tabular}{|c|c|c|c|c|c|c|c|c|}
\hline \multirow[b]{2}{*}{ Location } & \multirow{2}{*}{$\begin{array}{l}\text { Received HSUPA } \\
\text { signal (dBm) }\end{array}$} & \multirow{2}{*}{$\begin{array}{c}\text { GPS } \\
\text { Satellites } \\
\text { in View }\end{array}$} & \multicolumn{2}{|c|}{ Throughput (kbps) } & \multirow{2}{*}{$\begin{array}{c}\text { Latency } \\
\text { UCCAR-CSS } \\
\text { (ms) }\end{array}$} & \multicolumn{3}{|c|}{ Camera } \\
\hline & & & Download & Upload & & $\begin{array}{c}\text { Visualization } \\
\text { experience }\end{array}$ & $\begin{array}{l}\text { Bitrate } \\
\text { (Kbps) }\end{array}$ & $\begin{array}{c}\text { Qf } \\
\text { (bits/pixel·frame) }\end{array}$ \\
\hline 1 & -69 (Excellent) & 7 & 3200 & 1300 & 45 & Fluid, uncut & 787 & 0,0759 \\
\hline 2 & $-67 \quad$ (Excellent) & 7 & 3200 & 1400 & 44 & Fluid, uncut & 832 & 0,0802 \\
\hline 3 & (Good) & 7 & 2510 & 1265 & 45 & Fluid, uncut & 751 & 0,0724 \\
\hline 4 & $-79 \quad$ (Regular) & 9 & 2548 & 1356 & 44 & Fluid, uncut & 806 & 0,0777 \\
\hline 5 & (Poor) & 7 & 2702 & 1433 & 44 & Fluid, uncut & 851 & 0,0821 \\
\hline
\end{tabular}

Table 1. H.264 video transmission tests over HSUPA. 


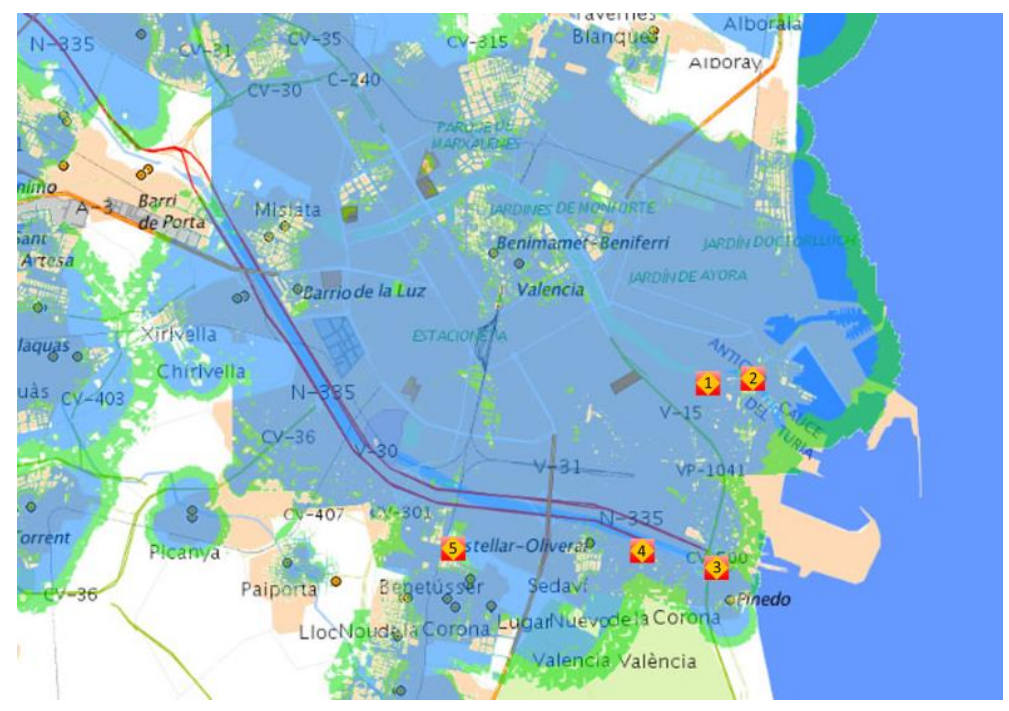

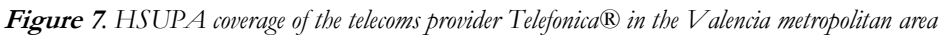

Table 1 shows the results of the tests carried out at the points marked in Fig. 7, which are considered by the operators to be points of special interest for monitoring purposes (for example, key points prone to flooding). The objective evaluation of the system's performance was done using frame quality. This parameter is a quality factor that represents the amount of bits used to encode each pixel in each frame in a video stream, and is calculated as:

$$
Q_{f}=b /(v \cdot h \cdot f)
$$

where $Q_{f}$ is the quality factor, $b$ is the bitrate (expressed in bit/s), $v$ is the vertical resolution of the frame, $b$ is the horizontal resolution of the frame, and $f$ is the frame rate (expressed in frames per second).

An important aspect of the test results to consider is that all locations obtained stable HSUPA coverage, and thus the transmission was fluid and without breaks, with small variations in quality. As can be seen in the results shown in Table 1, the latency (one-way) is constant at all the points, and is very low $(44 \mathrm{~ms})$, which has positive repercussions on camera control (turn, zoom), which is carried out practically in real time, without significant delays between ordering the camera movement and receiving the image. In relation to the uplink rates, it is worth highlighting that it is the worst HSUPA coverage that gives the best velocity ( $1433 \mathrm{kbps}$ with a signal of $-81 \mathrm{dBm}$ ). However, the power received is not the best indicator of the throughputs that will be achieved, as this depends mainly on the occupation rate and the backhaul bandwidth of the antenna to which it is connected, for example, location 5 is situated in an industrial zone. The buildings bring coverage problems, but these areas have greater data capacity.

We can also see that the video coding bitrate adapts dynamically to the available up-link flow, and that the quality remains between 0,072 y $0,082^{2}$. Thus, the coding and transmission in H.264 represents a significant saving in the quantity of bits necessary to code the pixel, which translates into an increase in quality using the same bandwidth. (Hanzo, Cherriman, \& Streit, 2007).

${ }^{2}$ HDTV via satellite has a quality factor between 5 and 6 , the DV between 1.8 and 2.4, the DVD between 0.4 and 0.8 and DivX between 0.2 and 0.4 . 

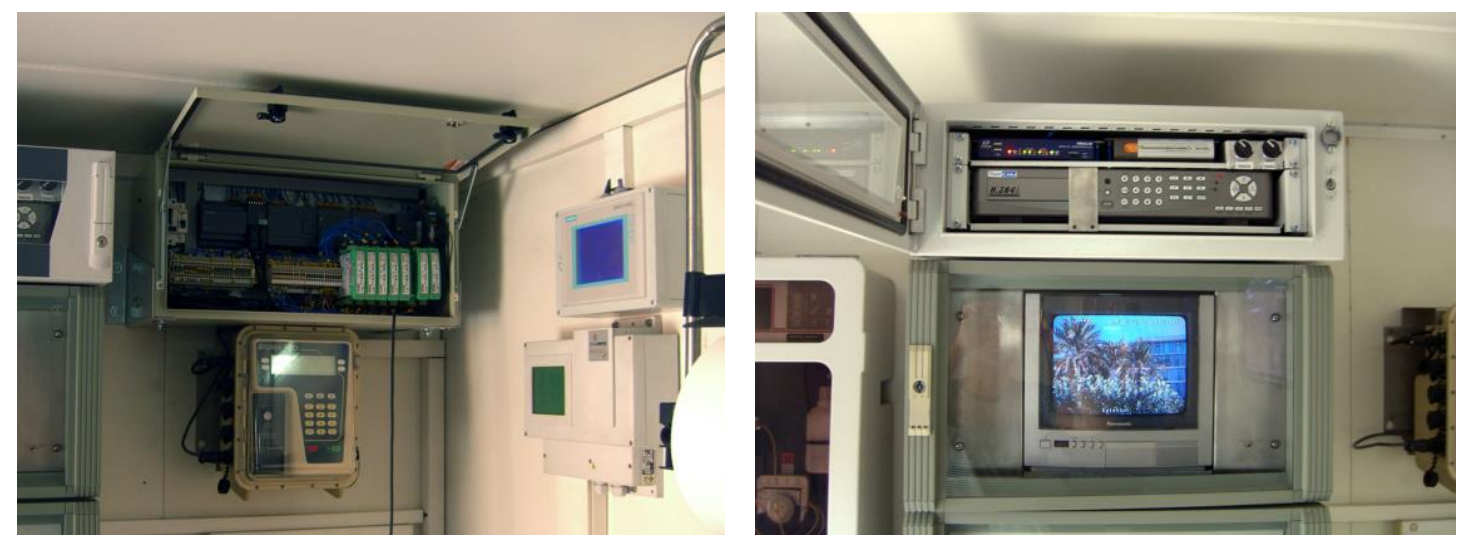

Figure 8. Details of the measurements (left) and vision (right) systems inside the UCCAR.

With the new vision system (fig.8, right), the operators can locate the UCCAR at a critical point in the network and monitor it from the CCS without the need for specialized personnel in the vehicle. In this way, vigilance of the point can be maintained longer, with automatic recording and warnings issued of changes in the image, enabling staff to make decisions in the crisis management group based on real time images of the problem. This system satisfactorily resolves problems of uncontrolled dumping and presence of solids in the network.

With the measurement sending system (fig.8, left), the water quality parameters obtained are centralized in the CCS with their geo-reference mark, enriching the measurement data from the fixed stations for less cost, and making it unnecessary to manually take the measurements, eliminating any possible human error which may occur.

\section{QDSN network architecture}

\subsection{Description}

A “Quick Deployment Sensor Network" (QDSN) is a collection of nodes which act as multi-purpose sensors and wireless routers, which can be deployed in a particular area, without any previous communications topology planning necessary, and which provides a series of tools developed to make their deployment agile and easy. This type of wireless sensor network (WSN) has a series of advantages in situations where only occasional measuring is necessary:

- It makes points accessible that were previously impossible due to cable problems.

- The network can be deployed and collected more quickly, with less equipment necessary, and without the need for specialist personnel.

- All the information collected by the network converges at a single node, which then communicates the information to the outside. There is less expense, as now a data tariff in each sensor node is not necessary.

- Greater distances can be achieved as the nodes in mid-route act as repeaters that extend the range.

With the QDSN it is possible to take measurements from zones where it is not viable to install permanent measuring points, allowing:

- Periodic control of spillways in industrial areas to detect nocturnal dumping.

- Environmental controls at various points around the city.

- Measuring of anomalous vibrations and temperatures in pumps and/or impulsion ducts.

- Flexible set up at variable points in the drainage network to measure parameters.

\subsection{Design}

The QDSN is made up of two types of elements: the nodes (fig. 9) and the Gateway. The nodes capture the measurements and then coordinate to deliver them to the Gateway. The Gateway acquires all the data and then sends it to the CCS. 


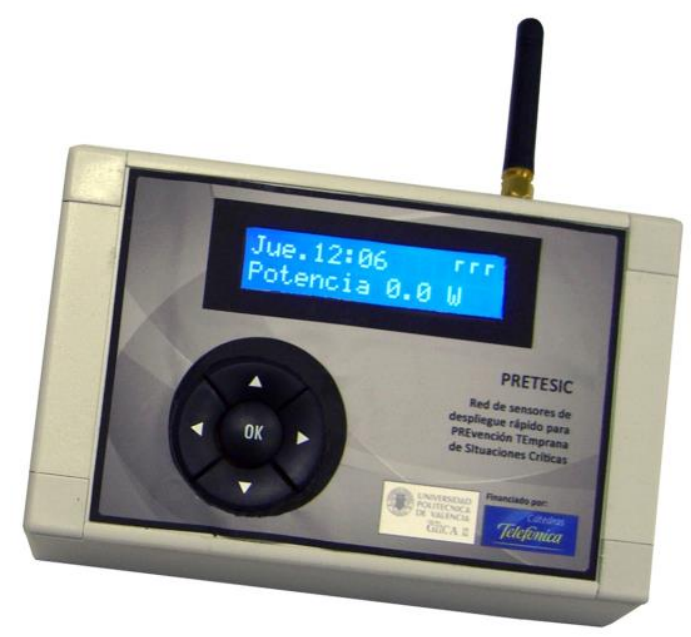

Figure 9. Detail of a QSDN node. Sensors are attached to it via connectors on the left side

Each of the elements has a size of $11 \times 4 \mathrm{~cm}$ and is made up of a board based on the micro ATMega, with an IEEE 802.15.4 communications module for the communication between nodes. Each measuring node has an ADC converter of 16 bits, with which extremely precise values are obtained from the probe it is connected to, which, depending on the application, could be a sensor for presence of liquids, phreatic level, temperature, energy, etc. This value is transmitted via I2C (Inter-Integrated Circuit) bus to the micro-controller to be processed. Once the sensor has carried out its function, the node must send the measurement data, for which it has two modes: local and remote.

For the local mode, a compact user interface has been incorporated, made up of an LCD screen of 16x2 characters, with a joystick and two buttons. This interface allows the user to consult the measurements taken and configure the system, as well as set up the sensors simply and easily.

For the remote mode, we designed our own application layer for the communication between the nodes in the network, with configuration and consultation of data messages. When the measuring node is consulted by the Gateway, it sends its most recent measurement. Moreover, the measuring nodes also operate as activators, such that from the CCS, they activate/deactivate devices connected to them. Communication between the network and the outside is done using the Modbus protocol, and so the system is compatible with other data acquisition and transport, evaluation and test elements ( $\mathrm{Yu}$, Liu, \& Wang, 2009). The nodes use an address of 5 bits, which allows a range of up to 32 addresses. As the addressing at the application level is done with Modbus addresses, and the 802.15.4 module uses its MAC addresses MAC (Media Access Control) for the network's internal communication, the Gateway must maintain an address translation table and act as a transparent link between the Modbus protocol, the outside and the QDSN. When a measurement data request reaches the Gateway, it is directed to a Modbus address. It is automatically translated to re-send the request to the corresponding node in the network. The assignment of a new address to a node in the network means there must be communication between the node and the Gateway to negotiate this change and keep the address translation table up-to-date. Moreover, network addresses are used to segment a large quantity of nodes in diverse networks. It is possible to use up to 12 networks in the same area, corresponding to each of the radio-frequency channels of the device, and each one will be made up of a Gateway and up to 32 measuring nodes. In this way, the nodes with addresses 3.15 and 2.06 will correspond respectively to node 15 of network 3 and node 6 of network 2, and each will communicate with different Gateways (the one responsible for network 3 and that of network 2).

\subsection{Operation}

As was seen in section 6.1., the concept of "quick deployment" means the network is set up quickly and without planning. This means an increase in complexity in the node, which must have protocols and tools allowing the user to set up the measuring nodes, check the coverage at each point, and advise the user of the validity of each point of interest. The working plan designed in the QDSN network is as follows: 


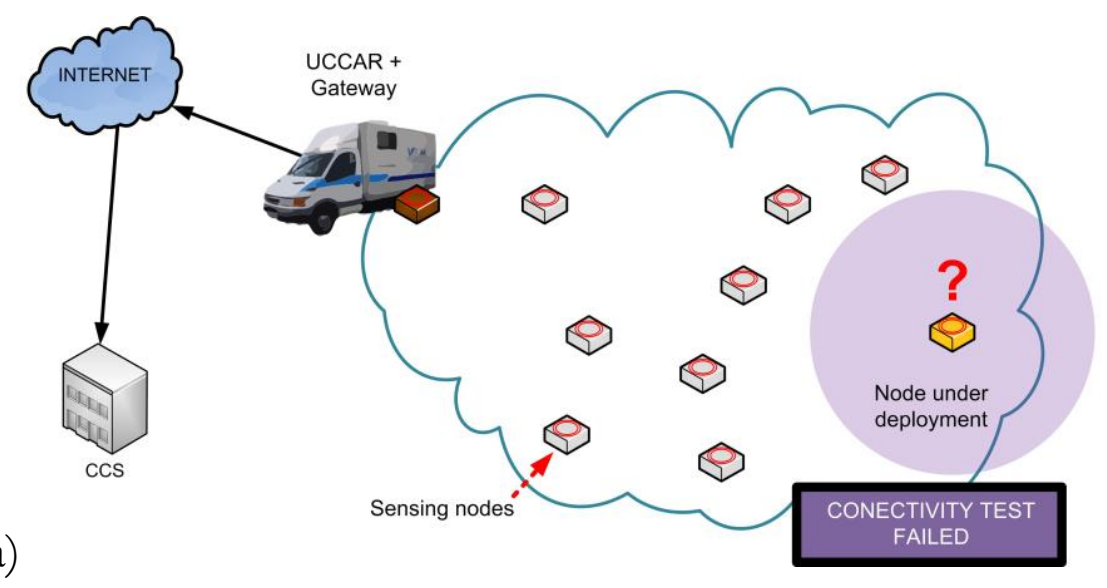

(a)

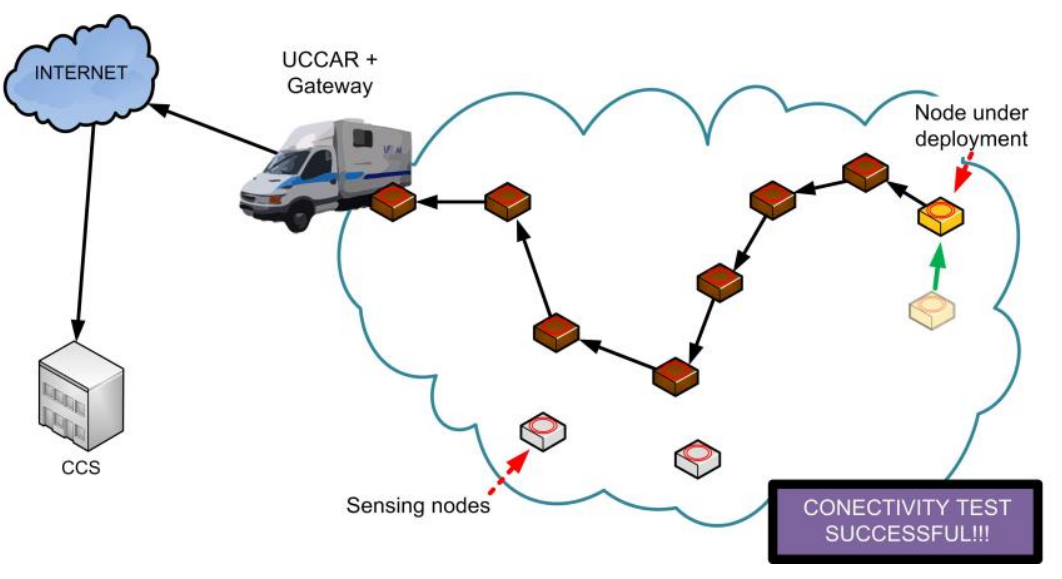

(b)

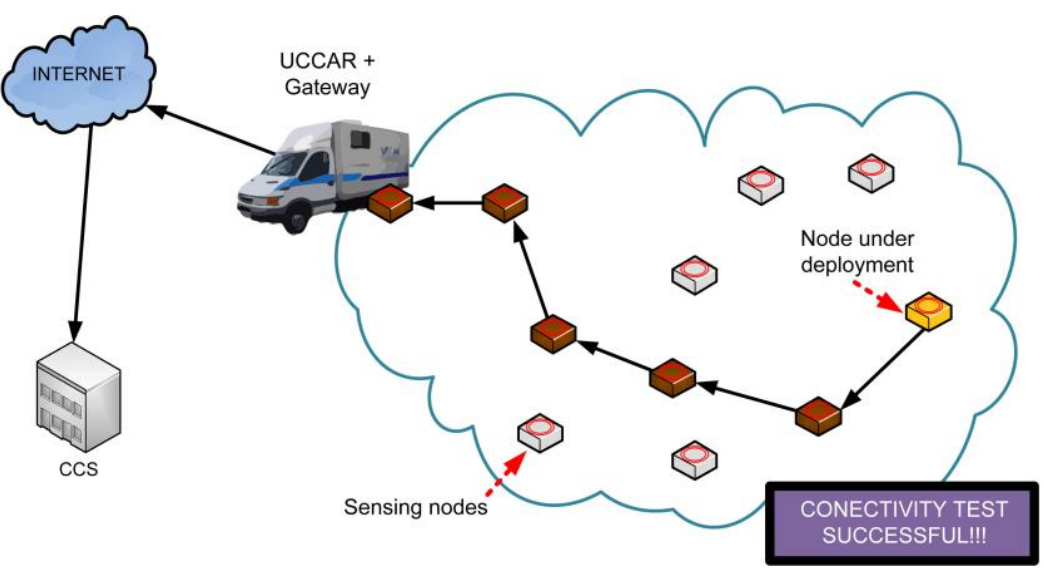

Figure 10. Adding a node in deployment phase.

1. Manual assignment of a network address to all the elements in the same network (all the nodes that communicate with the same Gateway must have the same network number, from 1 to 12).

2. The "Gateway discovery" tool. When this is executed from the node menu, it looks for the Gateway of its network, to learn its physical address (MAC). This address will be used for the communications initiated by the measuring node.

3. Manual assignment of the Modbus address of each node. It is the operator's responsibility to assign unique addresses to each measuring node within the same network. There must also be communication with the Gateway, as it must add the new node to its list and enter it to the address translation table.

At this point, the measuring node communicates with the Gateway, and can send its measurement. To help with the final location of the measuring node, the following tool was developed:

4. "Connectivity test" tool. Every time that this is executed in the menu, the measuring node tries to connect with the Gateway whichever way possible, either directly or via nodes that are distributed around the network. The multi-hop 
communications and the route search are transparent to the user, who will only see in the measuring node if the communication has been possible or not.

Fig.10 shows a node during the set up process. In its current position (Fig.10a) the node cannot reach any other node or the Gateway, and so the "Connectivity test" shows that there is no coverage. Fig.10b shows how the user has changed position and that now, the node has found a route to reach the Gateway using another measuring node, and so the "Connectivity test" shows as affirmative. In Fig. 10c the initial position of the node is maintained. As this point did not have coverage, a new node has been installed between this and another, and now there is coverage, thus establishing a new route to the Gateway.

The Gateway consults the nodes in order, and then sends the formatted data to the communication system of the UCCAR via Modbus over RS-485. This data is added to the cue, and is then sent to the CCS where it will be stored.

\subsection{Communications system}

To complete the functioning plan proposed, there must be coordination between the elements of the network, and the system must be totally flat (without hierarchy). If it is not, certain nodes with concrete functions would have to be located at specific points so that communication can take place, as is the case of the coordinator nodes in Zigbee (Wheeler, 2007) or of the access points, which would mean planning the network set-up. Due to this and other characteristics such as the constant re-location of nodes, the freedom of location of this equipment, or the existence of communications initiated in the central element (consultation of measurements) or in the final elements (Connectivity test, Gateway search and change of address), a wireless system of quick deployment cannot be supported by classic communication plans.

As a final solution, we used a wireless mesh sensor network design over IEEE 802.15.4 technology in ISM band of 2.4 GHz. With a transmission power of $100 \mathrm{~mW}$, for which the range surpasses one km outdoors and up to $300 \mathrm{~m}$ indoors, depending on the distribution of the building. With this topology, communication between nodes is ad hoc and multi-hop (mesh), and so each measuring node acts as a final node (sensor) and also as a router. The coordination of the nodes for route searches, when one of the measuring nodes wants to communicate with Gateway, or even when the Gateway itself wants to act on one of the measuring nodes, is done using a protocol based on a modification of the reactive protocol (route search on demand) AODV (Ad hoc On Demand Distance Vector) (Albero, Santonja, Sempere, \& Mataix, 2009). However, control of lost packets, access to the medium or re-sends are not resolved with this. As there is a central element, the Gateway, and the communication can be initiated by both the Gateway and the nodes, it may seem that the obvious solution is a token-passing protocol. However, its use would bring efficiency problems for two reasons:

- The token pass to all the nodes in the network generates constant additional route search traffic in all the nodes, as the protocol is a reactive routing one. This generates higher battery consumption and constant access to the medium by all the nodes, increasing the rate of collisions and re-sends.

- Communication initiated by the sensors is a special case, as it only happens during the network deployment process. In a network that is up and running, communication will only be initiated by the Gateway, which will consult the measurements from the nodes. Therefore, it would create even more problems to continue to generate and pass tokens through all the nodes in the network.

For this reason, we have opted for a traditional master-slave polling system for the communication initiated by the Gateway (Fischer, Laakonen, \& Schulz, 2001), in combination with an "Aloha" access to the medium for the communication initiated by the measuring nodes. We are referring to a "Polling + Aloha forced interruption", because if the Gateway is in the polling process at the moment when the node wants to start communication, it is almost certain that a collision will be produced. However, this collision is not a problem, but rather a way of advising that a node wants to initiate communication, and so this is a polling process interruption.

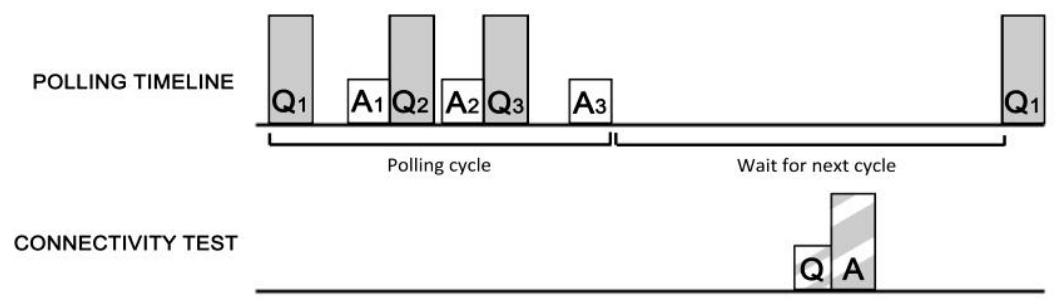

Fig. 11a: Connectivity test done in the waiting cycle of the Gateway 


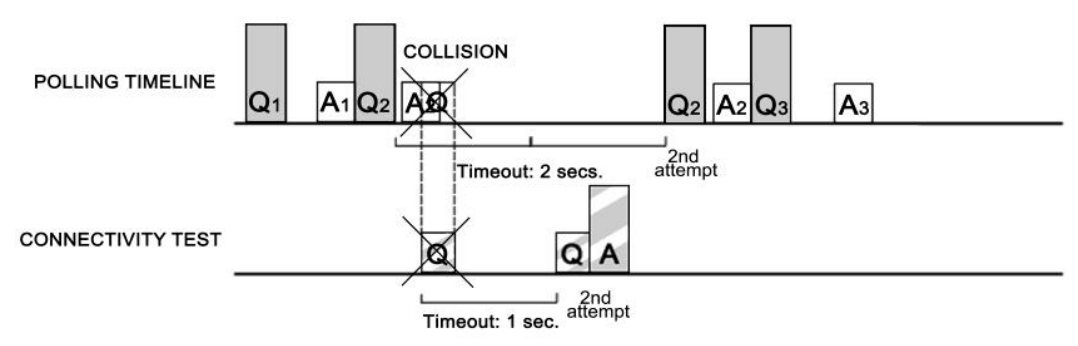

Fig. 11b: Connectivity test done in the polling cycle of the Gateway

In Fig.11a, the bigger bars represent the Gateway frames, and the smaller bars represent the frames from the nodes. In the polling timeline, we can see how during the polling cycle the server consults the measurements of nodes 1, 2 and 3 (Q1, Q2 and Q3), which respond after a short delay, (A1, A2, A3) due to their distance and the number of hops to the node being consulted. If an external node wants to carry out a connectivity test $(\mathrm{Q})$, this test will be successful if the consulting datagram connects to the medium when it is not busy, that is during the waiting times between polling cycles, or the response waiting time of a node to a consultation on its measurements.

The polling cycle is very fast when there are no problems of communication between nodes, meaning there is a strong probability of collisions if the connectivity test is done during this period. In Fig. 11b, the external node carries out a connectivity test during the polling cycle $(\mathrm{Q})$, which collides with the response of node $2(\mathrm{Q} 2)$, and at the same time the external node has initiated a timer of 1 second before the connectivity test $(\mathrm{Q})$. The external node's timer runs out and it re-sends its consultation (Q), to which the Gateway responds. After this, the polling cycle continues with a second consultation re-try to node 2 (Q2), which is successful (A2).

In this way, the nodes that are in the process of being deployed and set up have the capacity to interrupt the polling cycle. In noise free conditions, if the consultation is carried out during the waiting cycle, the response is instantaneous. If it is carried out during the polling cycle, there is a delay of 1 second. As was mentioned previously, the communication initiated by the nodes only happens during the deployment of the network. When the network is installed and operational, the communication will only consist of the polling cycles of the Gateway, avoiding the need for additional tools for access to the medium.

\subsection{Results}

Cost reduction: the QDSN does not need qualified personnel for its deployment and set-up meaning the same staff that operates the UCCAR to take various measurements can manage the QDSN. Costs are reduced as a single network can serve to carry out diverse measurements in diverse locations. Moreover, these measurements are transmitted from the communications system in the UCCAR, and so it is not necessary to use a public network with each of the nodes (as happens in the ECS stations).

Precision and reliability: Automation of the measurements acquisition process minimizes the possibility of human error, and these are centralized in the CCS without the need to handle or transport the data, meaning that precise measurements are obtained in situ and with greater frequency.

Speed: due to the design of the network, its deployment in critical zones at moments of risk allows the capture of values from the first moment, giving all the information necessary to act and predict accordingly.

\section{Architecture in the Central Control Station}

The CCS controls communications with the monitoring and activity stations, and is responsible for managing and processing all the information collected from the stations.

It is made up of different items of equipment (fig.12): a Central Control, a Web server, a Database server and a firewall, as well as communications equipment necessary for communication with the public network for external users and the private network that interconnects the stations via IP and a heterogeneous combination of wired and wireless communication technology. The processing and communication load is distributed over three pieces of equipment, as from the CCS the states of multi-media information and control elements are operated and accessed and this requires a greater processing capacity (Sempere, Albero, \& Silvestre, 2003). 


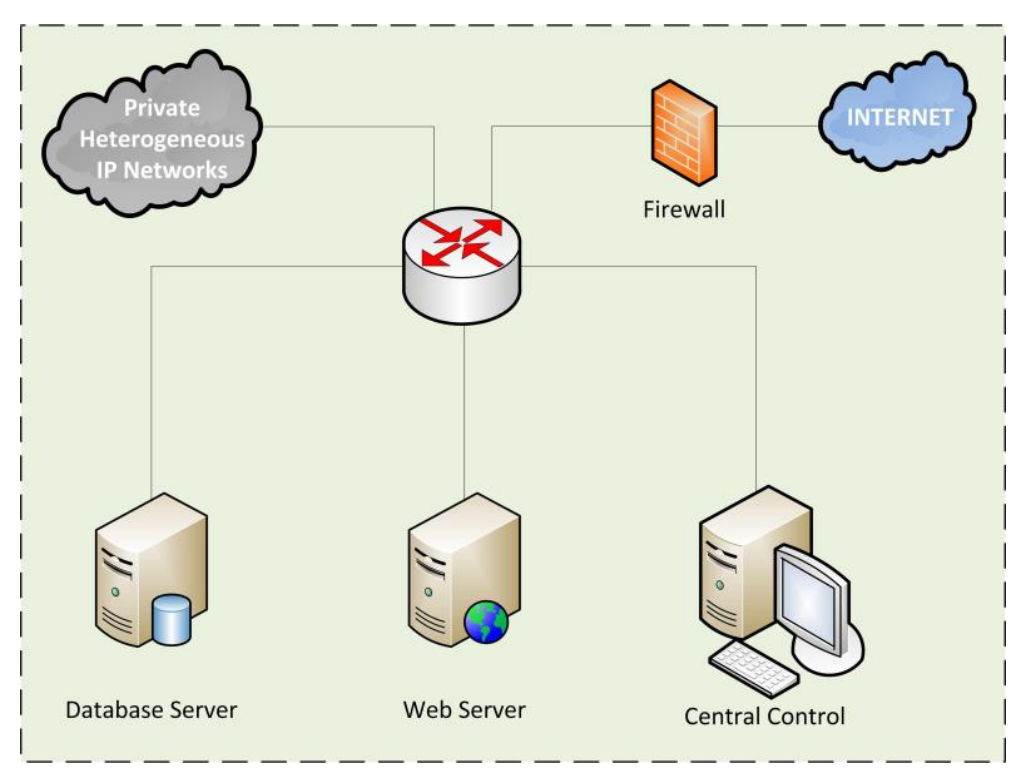

Footnote: Fig. 12: Central Control Station Scheme

In the equipment which houses the Control Station, the OPC Server (Ole for Process Control) is executed and this manages the connections with the remote stations. The connection server receives, processes, and stores the data in the database server. It also serves the OPC client, which acts as a user application from which the whole system is managed (state of the equipment in the remote measuring stations, consultation of values obtained by the sensors, real time configuration of remote equipment, set up, modification and un-subscription of stations and users, definition of threshold values for alerts and memory states, etc...). Internally, the connections server maintains an image of the memory space of the remote station controllers, and the CCS client accesses this memory space to modify the defined configuration variables. It is the task of the remote stations to keep this memory space up to date through the periodic sending of its measurements. The client reads this memory space continuously so that as new data arrives he can take the necessary action, such as sending an SMS to the person responsible for this area, alerting that a value that has passed a threshold.

The Web server, based on Apache, provides control information and images to the remotely connected users (Fig.13), allowing them to carry out their work instantaneously without the need to actually be in the installation. These users can connect from any IP device, whether it be computer, smartphone, laptop, or other device. The platform has special tools to deal with rainfall, and using different parameters and sensor points, can know the state of the system and the drainage capacity of the city at all times. There are different levels of security that decide which information is available to which user according to his security level.

The data base server works with the MySQL free software. This data is very important as it is used to take decisions on what action is necessary, and for this reason its availability and integrity are ensured through hardware redundancy and duplication of the data stored. This data can be accessed externally via the Web server or the CCS using SQL (Structured Query Language) consultations. The Firewall provides security against external attacks, and has been implemented with GNU/Linux using IPTables. Finally, both the local equipment and the remote stations incorporate the Simple Network Management Protocol (SNMP) to manage the network from the CCS. 

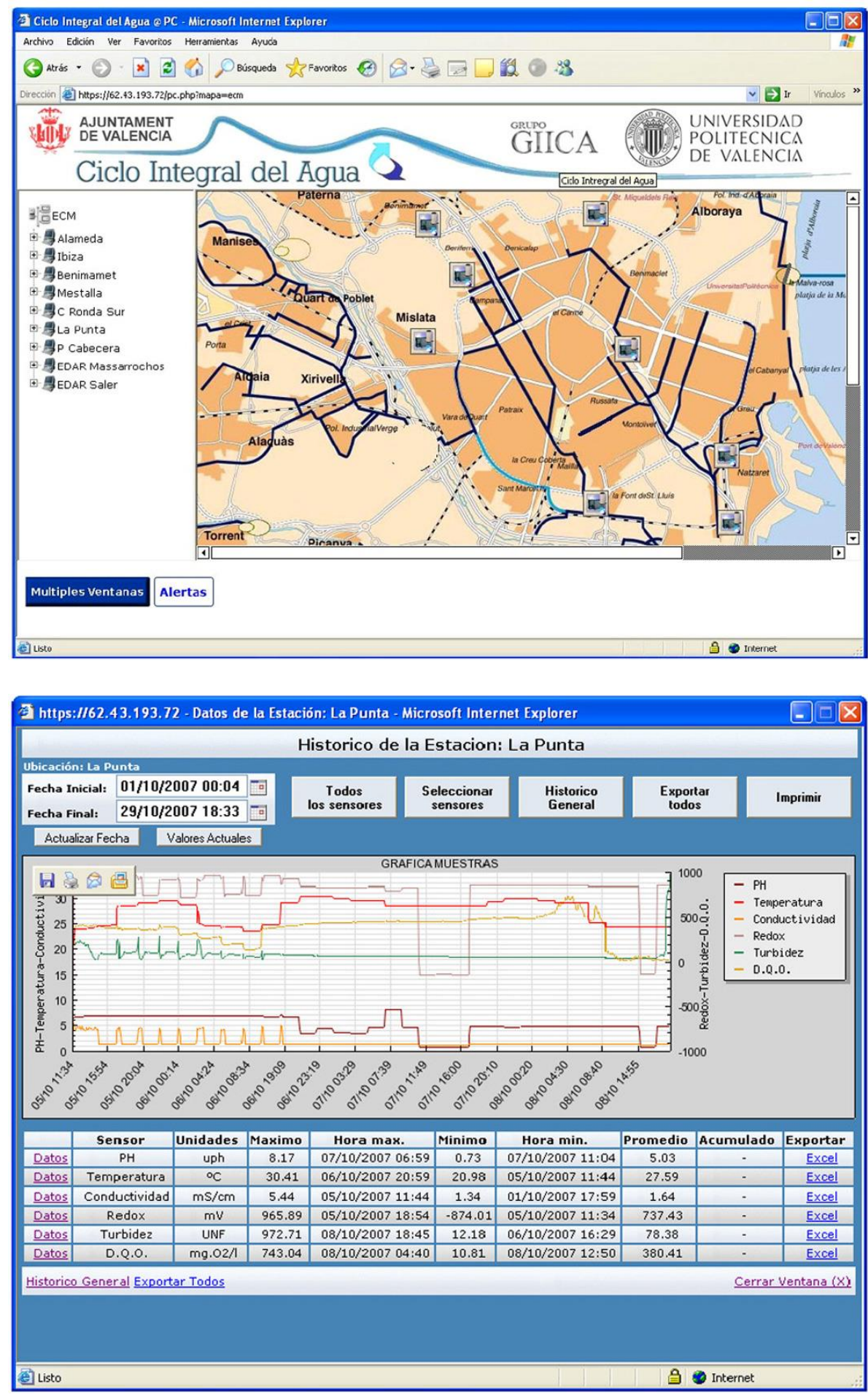


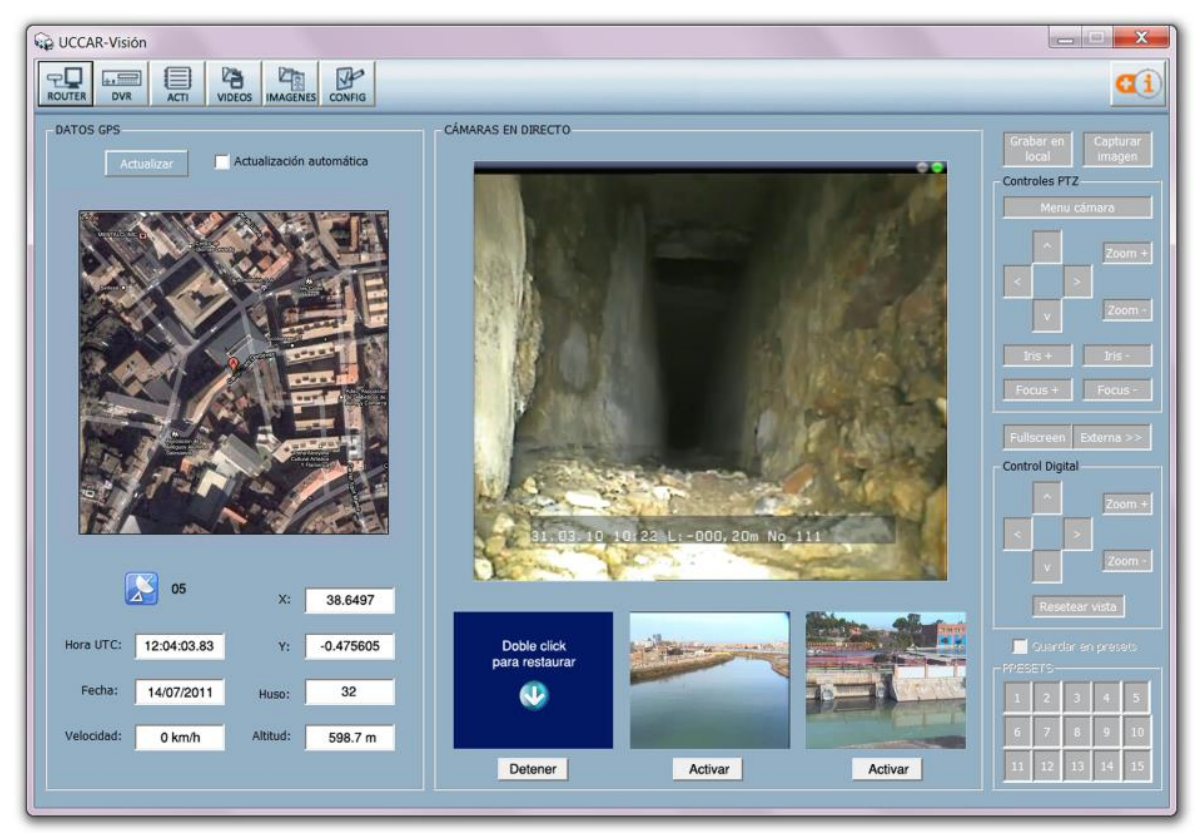

Figure 13. Detail of the web page used to consult parameters of the network, and vision system

\section{Conclusions}

The integration of information and communication technologies in Utilities Networks brings innate benefits for management of and access to information, making communication easier and more flexible, which in turn means the information collected is dealt with in a more advanced way. We have shown an example of the application of these technologies in the waste water system of Valencia, which, thanks to the improvements in the sensor system and the development of new communications systems, has given a powerful monitoring and control tool to the management team of the installation.

The new system gives high added value to the existing infrastructure, which facilitates and automates the management of parameters captured and the action that needs to be taken. In this way, the quality of information necessary for decision making is greatly improved. Data is provided with more precision, greater frequency, with historical and statistical relationships, and includes images, geo-references, enabling warnings to be sent of alarming situations.

For situations where only sporadic measurements are necessary, in places without infrastructure, at times of alert, such as control of dumping or measuring of isolated environmental parameters, the new QDSN allows the deployment of a measuring system that is instantaneous, fast and simple, avoiding the need to plan and set up a communications infrastructure. With these improvements, the system can be deployed by unqualified staff, perform measurements from the CCS, and change to the next measuring point in a short span of time, all of which greatly reduced costs.

With the new sensor system, all the data from the network flows to a single point, the CCS. There, the data is evaluated and stored, minimizing the human factor in the transport of data to the CCS, including the measurements of parameters carried out in the mobile laboratories (UCCAR). Altogether, this automation process minimizes errors with the data and increases its availability. This means that the technicians can dedicate a greater portion of their time to making decisions and taking action, rather than continuously collecting data for analysis.

The next steps towards this project focuses on merging the new concepts of "Internet of Things" and "Smart Cities" to the advanced management of urban sewer systems, providing semantic content for sensor networks, making them accessible from any external element through internet so data can be automatically acquired and processed, transparently to location. This will significantly increase the number of sensing points and facilitate management. The availability and universal nature of these contents will allow data sharing between institutions and remote locations, increasing the quality and quantity of information available for decision making, and the development of a public service with real-time information available to all residents. Ultimately, this will result in a more efficient management system, a safer city, and a population more involved in the use of water and city decisions.

\section{Acknowledgement}


This work was supported by the MCyT (Spanish Ministry of Science and Technology) under the projects PET2007-0316 and TIN2010-21378-C02-02, which are partially funded by ERDF (European Regional Development Fund).

\section{References}

ACQUEAU (2010). Public Information on Project Outlines, 9-10.

Albero, T., Santonja, S., Sempere, V. M., \& Mataix, J. (2009). AODV Performance Evaluation and Proposal of Parameters Modification for Multimedia Traffic on Wireless Ad hoc Networks. IFIP Wireless Days, 1-6.

Andritsou, A.I., \& Pronios, B. (2001). Mobility Convergence in Heterogeneous (Fixed and Mobile) Networks. Mobile Networks and Systems, 97-100.

Artell, T., Koivisto, H., Seppälä, J., \& Ruohonen, T. (2005). Quality of Service in Network-based Automation. Proceedings of the 16th IFAC World Congress.

Fischer, R.A., Laakonen, A. S., \& Schulz, N. N. (2001). A general polling algorithm using a wireless AMR system for restoration confirmation. IEEE Transactions on Power Systems, 312-316.

Granzer, W., Kastner, W., Neugschwandtner, G., \& Praus, F. (2006). A Modular Architecture for Building Automation Systems. IEEE Int. Workshop on Factory Communications Systems, 99-102.

Hanzo, L., Cherriman, P. J., \& Streit, J. (2007). Video compression and communications: from basics to H.261, H.263, H.264, MPEG4 for $D V B$ and HSDP A-style adaptive turbo-transceivers. John Wiley \& Sons.

Hopkinson, K., Roberts, G., Wang, X., \& Thorp, J. (2010). Quality-of-Service Considerations in Utility Communication Networks. IEEE Transactions on Power Delivery, 1465 - 1474.

Mayer, K., \& Taylor, K. (2002). An Embedded Device Utilizing GPRS for Communications. International Conference On Information Technology and Applications.

Morley, M. S., Bicik, J., Vamvakeridou-Lyroudia, L. S., Kapelan, Z., \& Savic, D. A. (2009). Neptune DSS: A Decision Support System for Near-Real Time Operations Management of Water Distribution Systems. Proceedings of the 10th International Conference on Computing and Control for the Water Industry, 249-255.

Ocampo-Martinez, C., Puig, V., Cembrano, G., Creus, R., \& Minoves, M. (2009). Improving water management efficiency by using optimization-based control strategies: the Barcelona case study. Water Science \& Technology: Water Supply, 9, 565-575.

Östmark, Å., Eliasson, J., Lindgren, P., Halteren, A., \& Meppelink, L. (2006). An Infrastructure for Service Oriented Sensor Networks. Journal of Computers, 1, 20-29.

Pyayt, A., Lang, B., Mokhov, I., \& Ozhigin, A. (2010). UrbanFlood, an early-warning system for climate induced disasters. Conference 5 th annual conference of the International Network of Storm Surge Barrier Managers St. Petersburg.

Ritchie, J. C., \& Cooper, C. M. (2001). Remote Sensing Techniques for determining water quality: Applications to TMDLs. TMDL Science Issues Conference, 367- 374.

Schierl, T., Wiegand, T., \& Kampmann, M. (2005). 3gpp compliant adaptive wireless video streaming using h.264/avc. IEEE International Image Processing, ICIP, 3, 696-699.

Schütze, M., Campisano, A., Colasc, H., Schilling, W., \& Vanrollegheme, P.A. (2004). Real time control of urban wastewater systems - where do we stand today?. Journal of Hydrology, 299, 335-348.

Sempere, V.M., Albero, T., \& Silvestre, J. (2006). Analysis of communication alternatives in a heterogeneous network for a supervision and control system. Computer Communications, 29, 1133-1145.

Sempere, V. M., Albero, T., \& Silvestre, J. (2003). Supervision and Control System of Metropolitan Scope Based on Public Communication Networks. IFAC Fieldbus Technology and their Applications, 317-324.

Stockhammer, T., \& Hannuksela, M. M. (2005). H.264/avc video for wireless transmission. IEEE Wireless Communications, 6-13.

Suñer, D., Malgrat, P., Leitão, P., \& Clochard, B. (2008). COWAMA (Coastal Water Management) Integrated and Real Time Management System of Urban Water Cycle to Protect the Quality of Bathing Waters. 11th International Conference on Urban Drainage.

Thomesse, J. P. (2005). Fieldbus Technology in Industrial Automation. Procedings of the IEEE, 6, 1073-1101.

Verworn, H. R. (2002). Advances in urban-drainage management and flood protection. Philosophical Transactions of The Royal Society, 360, 1451-1460.

Wheeler, A. (2007). Commercial Applications of Wireless Sensor Networks Using ZigBee. IEEE Communications Magazine, 45, 70-77.

Wiegand, T., Sullivan, G. J., Bjntegaard, G., \& Luthra, A. (2003). Overview of the h.264/avc video coding standard. IEEE Transactions on Circuits and Systems for Video Technology, 13, 560-576.

Yu, C., Liu, Y., \& Wang, C. (2009). Research on ZigBee Wireless Sensors Network Based on ModBus Protocol. Wireless Sensor Networks, 1, 1-60. 